\title{
fabH deletion increases DHA production in Escherichia coli expressing Pfa genes
}

\author{
Laura Giner-Robles, Beatriz Lázaro, Fernando de la Cruz* and Gabriel Moncalián*
}

\begin{abstract}
Background: Some marine bacteria, such as Moritella marina, produce the nutraceutical docosahexaenoic acid (DHA) thanks to a specific enzymatic complex called Pfa synthase. Escherichia coli heterologously expressing the pfa gene cluster from M. marina also produces DHA. The aim of this study was to find genetic or metabolic conditions to increase DHA production in E. coli.

Results: First, we analysed the effect of the antibiotic cerulenin, showing that DHA production increased twofold. Then, we tested a series of single gene knockout mutations affecting fatty acid biosynthesis, in order to optimize the synthesis of DHA. The most effective mutant, fabH, showed a threefold increase compared to wild type strain. The combination of cerulenin inhibition and fabH deletion rendered a 6.5-fold improvement compared to control strain. Both strategies seem to have the same mechanism of action, in which fatty acid synthesis via the canonical pathway (fab pathway) is affected in its first catalytic step, which allows the substrates to be used by the heterologous pathway to synthesize DHA.
\end{abstract}

Conclusions: DHA-producing E. coli strain that carries a fabH gene deletion boosts DHA production by tuning down the competing canonical biosynthesis pathway. Our approach can be used for optimization of DHA production in different organisms.

Keywords: Fatty acid synthesis, Polyunsaturated fatty acid, Docosahexaenoic acid, Cerulenin, FabH

\section{Background}

Consumption of long-chain polyunsaturated fatty acids of the omega-3 series (LC-PUFA3) has been associated to benefits for human health [1]. LC-PUFA3 are found in membrane phospholipids and are especially abundant in neuron and retina. LC-PUFA3 play a major impact during fetal development, as well as in Alzheimer's disease progression [1]. Furthermore, they reduce chronic inflammation, which is the cause of many cardiovascular diseases [2]. Since only limited amounts of LC-PUFA3 are synthesized in humans from $\alpha$-linolenic acid (the main LC-omega-3 FA in vegetables), the Food and Agriculture Organization [3] recommends a minimum intake of $250 \mathrm{mg}$ LC-PUFA3 per day, while pregnant women

\footnotetext{
*Correspondence: fernando.cruz@unican.es;

gabriel.moncalian@unican.es

Departamento de Biología Molecular, Universidad de Cantabria

and Instituto de Biomedicina y Biotecnología de Cantabria (IBBTEC), CSIC-

Universidad de Cantabria, C/ Albert Einstein 22, 39011 Santander, Spain
}

intake must be $300 \mathrm{mg} /$ day, of which $200 \mathrm{mg}$ should be docosahexaenoic acid (DHA). However, the recommended daily intake is not reached in many countries, where consumption of fish and seafood is insufficient.

Currently, the main source of supplemented LC-PUFA3 is fish oil. Problems arise in the industrial production of LC-PUFA3, such as elevated purification costs, low stability of the molecules, undesirable odors, flavors and tastes, or the presence of a wide range of contaminants, including heavy metals. Furthermore, concerns about the sustainability of fisheries have increased the efforts towards alternative production sources $[4,5]$.

Besides fish, animals, plants, fungi and microalgae synthesize LC-PUFA3 from $\alpha$-linolenic acid by consecutive elongation and desaturation reactions. However, thraustochytrids and a group of deep-sea bacteria are able to produce de novo LC-PUFA3 by a multi-enzymatic protein complex named polyunsaturated fatty acid synthase (Pfa) [6]. The mechanism has not been characterized in detail, but is thought to be similar to Type II polyketide 
synthases (PKS) and de novo fatty acid synthases (FAS). LC-PUFA3 synthesis begins by the condensation of a malonyl group bound to an acyl-carrier protein (ACP) with an acetyl-CoA. This reaction is carried out by a $\beta$-ketoacyl-ACP synthase domain (KS). Later, there are several cycles of reduction, dehydration, reduction and condensation, to produce a 20-carbon long chain with five double bonds (C20:5, eicosapentaenoic acid, EPA) or a 22-carbon long chain with six double bonds (C22:6, DHA) [7, 8]. Orikasa et al. [7] cloned the five essential genes for DHA production from marine bacteria Moritella marina into an E. coli expression vector. The resulting plasmid, named pDHA4 (Table 1), contains $M$. marina pfaABCD genes under control of native promoters, while $p f a E$ is controlled by a T7 promoter. E. coli strain DH5 $\alpha$ containing pDHA4 produced 3.7\% DHA of total FAs after $96 \mathrm{~h}$ at $15{ }^{\circ} \mathrm{C}$ [7].

DHA production can be improved by treatment with sub-lethal concentrations of cerulenin. This antibiotic increased DHA production in M. marina [9], Colwellia psycherythraea [10], Thraustrochytrium sp. [11], and even in E. coli expressing the heterologous Pfa system [7, 12]. Pfa synthase is similar to Fatty Acid Synthase (FAS), responsible for short and medium chain fatty acid biosynthesis and uses the same substrates, malonyl-CoA and acetyl-CoA. In E. coli, de novo FAS-based FA synthesis uses three KS enzymes named FabB, FabF and FabH, which initiate FA elongation cycle. Although the three KS enzymes are structurally similar and participate in a similar reaction, the antibiotic cerulenin inhibits FabB and FabF, but not FabH [13, 14]. Thus, inhibition of FabB and FabF by cerulenin decreases the amount of substrate consumed by FAS, increasing by 50 -fold the intracellular level of malonyl-CoA available for heterologous synthetic pathways [15].

In this article we tested single gene in-frame knockouts in $E$. coli to find genetic modifications which improve the DHA production by Pfa heterologous system from M. marina. In particular, we found that $f a b H$ deletion produced a threefold improvement compared to the wild type reference strain. This modification could be used in oleaginous DHA-producing microbial species to enhance the production of this added-value fatty acid.

\section{Results \\ Cerulenin enhances DHA production in E. coli expressing M. marina pfa gene cluster}

The positive effect of the antibiotic cerulenin on the production of DHA in marine bacteria was previously known. DHA-producer $M$. marina treated with sublethal concentrations of cerulenin $(0.5-2 \mu \mathrm{g} / \mathrm{ml})$ showed a threefold increase (6-19\%) in DHA production [9], while Colwellia psycherythraea treated with $12 \mu \mathrm{g} / \mathrm{ml}$ cerulenin showed a fourfold increase in DHA content, accumulating up to $10 \%$ of total FA [10]. However, little is known about genetic modifications enhancing this production.

To study genetic effects on DHA production we have used the KEIO collection [16], a collection of single-gene deletions of all nonessential genes in E. coli K-12. First, we transferred pDHA4 to E. coli strain BW27783 (BWpDHA4 from here), a derivative strain from KEIO control strain BW25113 suitable for future assays with pBAD33 expression system, in which arabinose transporter and arabinose degradative enzymes have been modified [17-19]. BW-pDHA4 showed an increased production in DHA in $72 \mathrm{~h}$ when compared to $E$. coli DH5 $\alpha$ reported by Orikasa et al. [7] (from 3.7 to 7.5\% DHA of total FA).

We have first determined the optimal culture time and temperature for DHA production using BW-pDHA4 (Additional file 1: Fig. S1). We tested DHA production at different culture times obtaining $4.7 \%$ DHA/total FA at mid-exponential phase $(24 \mathrm{~h}), 7.0 \%$ at the end of exponential phase $(48 \mathrm{~h})$, and $4.6-5.1 \%$ during stationary phase (up to $96 \mathrm{~h}$ ). In addition, we tested DHA content at late exponential phase in cultures at different temperatures $\left(7.1 \%\right.$ DHA/total FA at $15{ }^{\circ} \mathrm{C}, 3.5 \%$ at $20{ }^{\circ} \mathrm{C}$, and $0 \%$ at $25^{\circ} \mathrm{C}$ ). Therefore, we established that the best conditions to produce DHA using BW-pDHA4 strain were $48 \mathrm{~h}$ at $15^{\circ} \mathrm{C}$.

Afterwards, we studied the enhancement of DHA production in the presence of different concentrations of

Table 1 List of $E$. coli strains and plasmids used in this work

\begin{tabular}{|c|c|c|}
\hline & Relevant characteristics & References \\
\hline \multicolumn{3}{|l|}{ Strains } \\
\hline BW25113 & F-, DE(araD-araB)567, lacZ4787(del)::rrnB-3, LAM-, rph-1, DE(rhaD-rhaB)568, hsdR514 & [16] \\
\hline BW27783 & 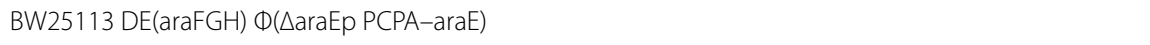 & [19] \\
\hline \multicolumn{3}{|l|}{ Plasmids } \\
\hline pDHA4 & $\begin{array}{l}\text { pSTV29 (Takara Corp.) carrying pfaABCDE from M. marina MP-1 (p15A OriV, } \sim 10 \text { copies, chlorampheni- } \\
\text { col resistance cassette) } \\
\text { pfaABCD expression is controlled by native M. marina promoters, pfaE is controlled by T7 promoter }\end{array}$ & [7] \\
\hline
\end{tabular}


cerulenin. As shown in Fig. 1, DHA (C22:6n3) content in BW-pDHA4 increased from 7.5 to $12.5,16.1$ or $17.0 \%$ in the presence of $0.5,1$ or $2 \mu \mathrm{g} / \mathrm{ml}$ cerulenin, respectively. Even though BW-pDHA4 achieved the highest DHA content at a cerulenin concentration of $2 \mu \mathrm{g} / \mathrm{ml}$, we performed the subsequent experiments using $1 \mu \mathrm{g} / \mathrm{ml}$ since the DHA (C22:6n3) content is almost similar at 1 or $2 \mu \mathrm{g} /$ $\mathrm{ml}$ and we had observed that high concentrations of cerulenin affected cell growth (Additional file 1: Fig. S2).

Cerulenin also had an effect on the overall FA composition in BW-pDHA4 (Fig. 1). Medium-chain saturated fatty acid content such as miristic acid (C14:0) increases in presence of cerulenin, while levels of palmitic acid (C16:0), palmitoleic acid (C16:1) and C17 cyclopropane acid $(\mathrm{C} 17 \mathrm{cyc})$ have not significant variations. In contrast, cis-vaccenic acid (C18:1n7) content drastically decreases. Similar variations in the fatty acid profile were reported for M. marina [9], C. psycherythraea [10] or E. coli expressing C. psycherythraea pfaABCDE genes [12].

\section{DHA production is increased in $\triangle \mathrm{fabH}$ mutant}

Cerulenin was shown to inhibit FabB and FabF, two main KS synthases involved in conventional FA synthesis [14]. Both enzymes are responsible for condensing malonyl-ACP with the FA growing chain. Besides FabB and FabF, FabH is the KS enzyme responsible of the initial condensation of malonyl-ACP to either acetylCoA or propionyl-CoA. However, FabH is not inhibited by cerulenin [20]. Thus, we hypothesized that, similar to the inhibition of FabF and FabB by cerulenin, deletion of $\mathrm{fabH}$ could also lead to an increase of DHA production. To test the $f a b H$ deletion phenotype, we transformed a $f a b H$ knockout mutant strain from the KEIO collection [16] with pDHA4 ( $\triangle$ fabH-pDHA4 from here). In Fig. 2a, we can observe a substantial improvement of DHA production caused by $f a b H$ deletion, reaching $24 \%$ DHA relative to total FAs. This level of DHA production implies a threefold improvement compared to BW-pDHA4, and corresponds to $11.2 \mathrm{mg} \mathrm{DHA} / \mathrm{L}$ (Table 2). Additionally, the absence of FabH activity altered the FA composition. Comparing the FA profile in Fig. 2a between BW-pDHA4 and $\triangle \mathrm{fabH}$-pDHA4 strains, we observe a major decrease in $\mathrm{C} 16: 0$ (36.5\% BW-pDHA4 vs $16.7 \% \Delta$ fabH-pDHA4), and $\mathrm{C} 17$ cyc $(15.4 \%$ vs $3.8 \%)$. In contrast, there is a significant increase in long-chain FAs, such as C18:1n7 $(23.5 \%$ vs $45.2 \%)$, as well as the already mentioned increment in DHA. Furthermore, $\mathrm{fabH}$ deletion had an impact on cell growth (Fig. $2 \mathrm{~d}$ and Table 2). Cells carrying this deletion ( $\triangle \mathrm{fabH}$-pDHA4) had a slower growth and thus a lower doubling time $\left(\mathrm{D}_{\mathrm{T}}\right.$, Table 2$)$ than reference strain BW-pDHA4 (5.3 h in BW-pDHA4 vs. $7.2 \mathrm{~h}$ in $\Delta$ fabH-pDHA4).

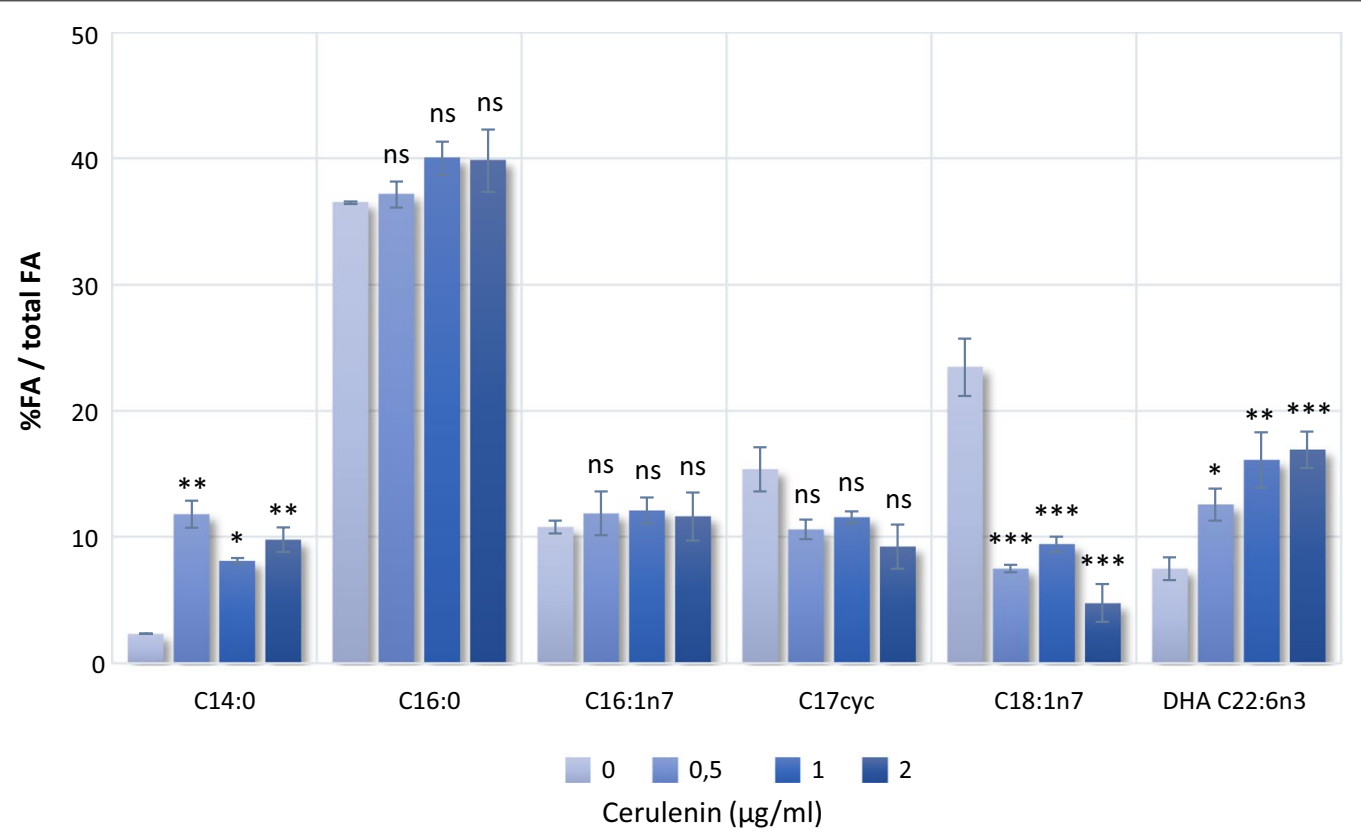

Fig. 1 Composition of major fatty acids in E. coli BW 27783 (pDHA4) treated with different concentrations of cerulenin $(0-2 \mu \mathrm{g} / \mathrm{ml})$ at $15^{\circ} \mathrm{C} \mathrm{for}$ $72 \mathrm{~h}$. Bars indicate the mean and standard deviation of the percentage of each FA in relation to total FA. Statistical analysis for each FA was made individually for at least three replicates using One-Way ANOVA. Asterisks indicate the degree of significance $\left({ }^{* * *} p<0.0001 ;{ }^{* *} p<0.001 ;{ }^{*} p<0.05 ; n s\right.$ not significant $\mathrm{p}>0.05$ ). Miristic acid —C14:0, palmitic acid-C16:0, palmitoleic acid—C16:1, C17 cyclopropane derivative-C17cyc, cis-vaccenic acid-C18:1n7, DHA—C22:6n3 

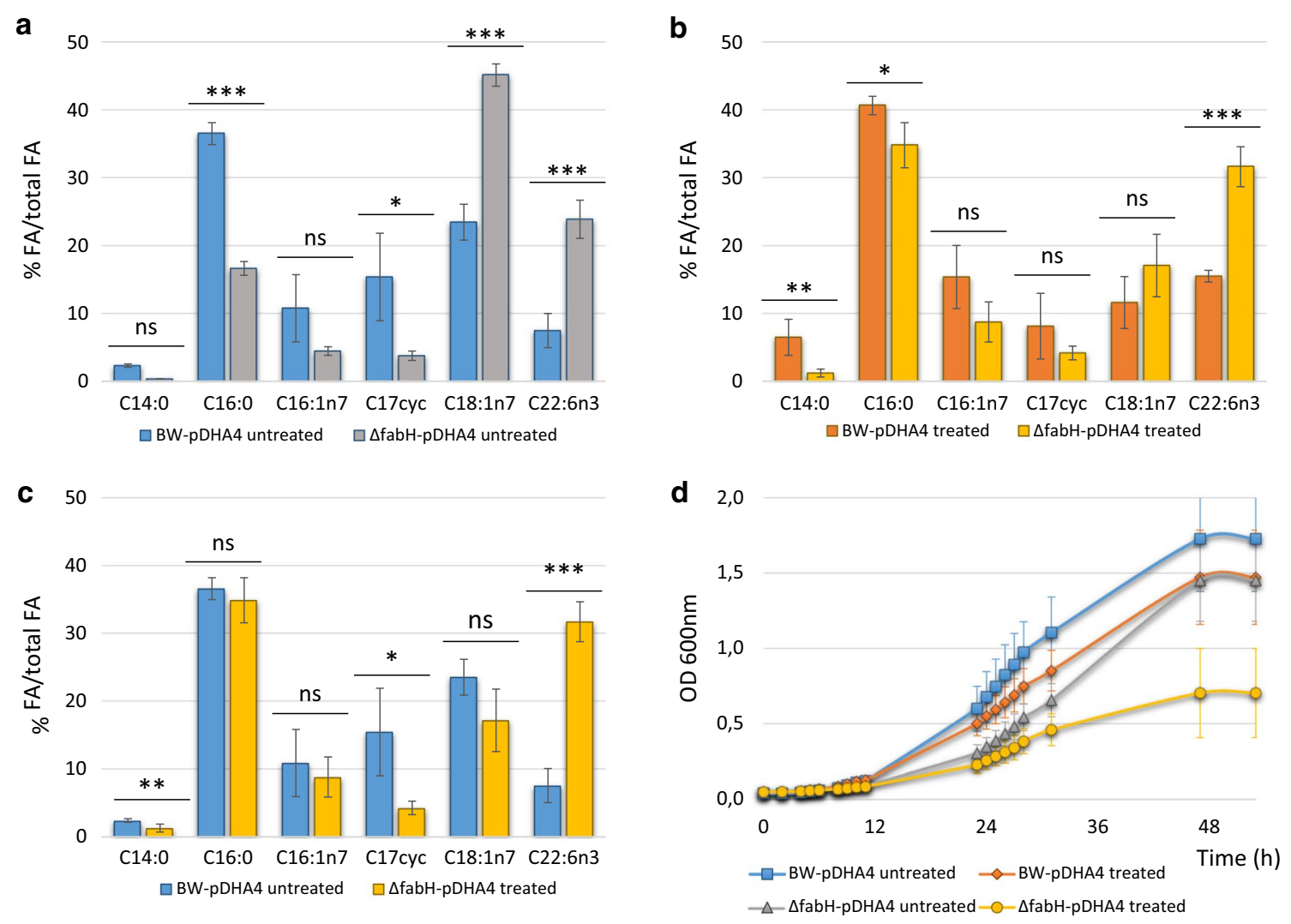

Fig. 2 Effect of $\mathrm{fabH}$ deletion and cerulenin on heterologous DHA production in E. coli strains cultured at $15^{\circ} \mathrm{C}$ for $72 \mathrm{~h}$. a Effect of fabH deletion on fatty acid composition of BW-pDHA4 and $\triangle \mathrm{FabH}$-pDHA4 in absence of cerulenin (untreated strains). $\mathbf{b}$ Effect of cerulenin on BW-pDHA4 and $\triangle \mathrm{FabH}-\mathrm{pDHA} 4$ in presence of $1 \mu \mathrm{g} / \mathrm{ml}$ cerulenin (treated strains). $\mathbf{c}$ Additive effect of fabH deletion and cerulenin treatment on fatty acid composition. Fatty acids from $\triangle \mathrm{fabH}$-pDHA4 treated with $1 \mu \mathrm{g} / \mathrm{ml}$ cerulenin are compared to fatty acids of untreated BW-pDHA4. Mean and standard deviation of each FA in relation to total FA content is shown. Statistical analysis was made for at least three replicates using One-Way ANOVA. Asterisks indicate the degree of significance $\left({ }^{* * *} p<0.0001 ;{ }^{* *} p<0.001 ;{ }^{*} p<0.05 ;\right.$ ns not significant $\left.p>0.05\right)$. Miristic acid-C14:0, palmitic acid-C16:0, palmitoleic acid—C16:1, C17 cyclopropane derivative—C17cyc, cis-vaccenic acid—C18:1n7, DHA—C22:6n3. d Growth curve of BW-pDHA4 and $\triangle \mathrm{FabH}-\mathrm{pDHA} 4$ with 0 and $1 \mu \mathrm{g} / \mathrm{ml}$ of cerulenin. Each curve was measured for $52 \mathrm{~h}$ at $15^{\circ} \mathrm{C}$ using 12 independent cultures

Table 2 Effect of $\mathrm{fabH}$ deletion and cerulenin inhibition $(1 \mu \mathrm{g} / \mathrm{ml})$ on DHA producing strains

\begin{tabular}{lllll}
\hline Strain & \multicolumn{2}{l}{ BW-pDHA4 } & \multicolumn{2}{l}{$\boldsymbol{\Delta \text { fabH_pDHA4 }}$} \\
\hline Cerulenin & $0 \mu \mathrm{g} / \mathrm{ml}$ & $1 \mu \mathrm{g} / \mathrm{ml}$ & $0 \mu \mathrm{g} / \mathrm{ml}$ & $1 \mu \mathrm{g} / \mathrm{ml}$ \\
Doubling time $(\mathrm{h})^{\mathrm{a}}$ & $5.3 \pm 0.2$ & $6.2 \pm 0.2^{* * *}$ & $7.2 \pm 0.2^{* * *}$ & $8.8 \pm 0.7^{* * *}$ \\
\%DHA/total FA $^{\mathrm{b}}$ & $7.5 \%$ & $15.5 \%^{* *}$ & $23.9 \%^{* * *}$ & $31.7 \%^{* * *}$ \\
mg DHA/l $^{\mathrm{b}}$ & $2.8 \pm 0.4$ & $8.1 \pm 0.24^{* * *}$ & $11.2 \pm 1.9^{* * *}$ & $16.8 \pm 1.6^{* * *}$
\end{tabular}

${ }^{a}$ Maximum doubling time calculated during the exponential phase for each condition

${ }^{\text {b }}$ DHA production is represented by relative (\% DHA/total FA) and absolute (mg DHA per liter of culture) quantification of DHA for each strain and condition Mean and standard deviation is shown. Asterisks indicate the degree of significance $\left.{ }^{* * *} p<0.0001 ; * * 0.001\right)$
In order to observe the effect of a tighter inhibition on the system, we analysed the effect of cerulenin on $\Delta$ fabH-pDHA4 cells. In this experiment, FA production was altered simultaneously by $f a b H$ deletion and by FabB and FabF partial inhibition with cerulenin. From the data in Fig. 2b, we can see that, under these doubly limiting conditions, DHA production reached 31.7\% of total FAs (16.8 mg DHA/L), 4.9-fold higher than BW-pDHA untreated. The proportion of some other FA species in $\triangle \mathrm{fabH}$-pDHA4 treated with cerulenin also changed compared to untreated BW-pDHA (Fig. 2c). For instance, the proportion of miristic acid in $\triangle \mathrm{fabH}$-pDHA4 treated with cerulenin $(2.3 \%)$ is significantly lower than in treated $\Delta \mathrm{fabH}-\mathrm{pDHA} 4(1.2 \%)$ 
while $\mathrm{C} 17$ cyc is reduced from $15.4 \%$ in untreated $\mathrm{BW}$ pDHA4 to $4.2 \%$ in treated $\triangle$ fabH-pDHA4. In relation to cell growth, this double inhibited system showed a low growth (Fig. $2 \mathrm{~d}$ ) with a $\mathrm{D}_{\mathrm{T}}$ of $8.8 \mathrm{~h}$ (Table 2).

\section{Effect of other KO mutants on DHA production}

To check the effect of other FA-synthesis mutations of the KEIO collection, as well as to stablish a control for the effect of in-frame deletions, we tested DHA production in a series of KEIO mutants transformed with pDHA4 (Fig. 3). The following mutants were selected (Table 3): i. Three non-essential genes related to FA biosynthesis besides $f a b H$ : $f a b F$ (ketosynthase responsible for cisvaccenic production), fabR (global regulator of fatty acid metabolism) and tes $A$ (FA thioesterase). These three mutant strains, transformed with pDHA4, did not show a significant improvement in DHA production compared to control strain BW-pDHA4. As shown in Fig. 3, DHA content increased from 7.5\% in BW-pDHA4 to $10.9 \%$ in $\triangle f a b R$-pDHA4 and $12.5 \%$ in $\triangle$ tesA-pDHA4 under the same culture conditions. Curiously, the single deletion of $f a b F(\triangle \mathrm{fabF}-\mathrm{pDHA} 4)$

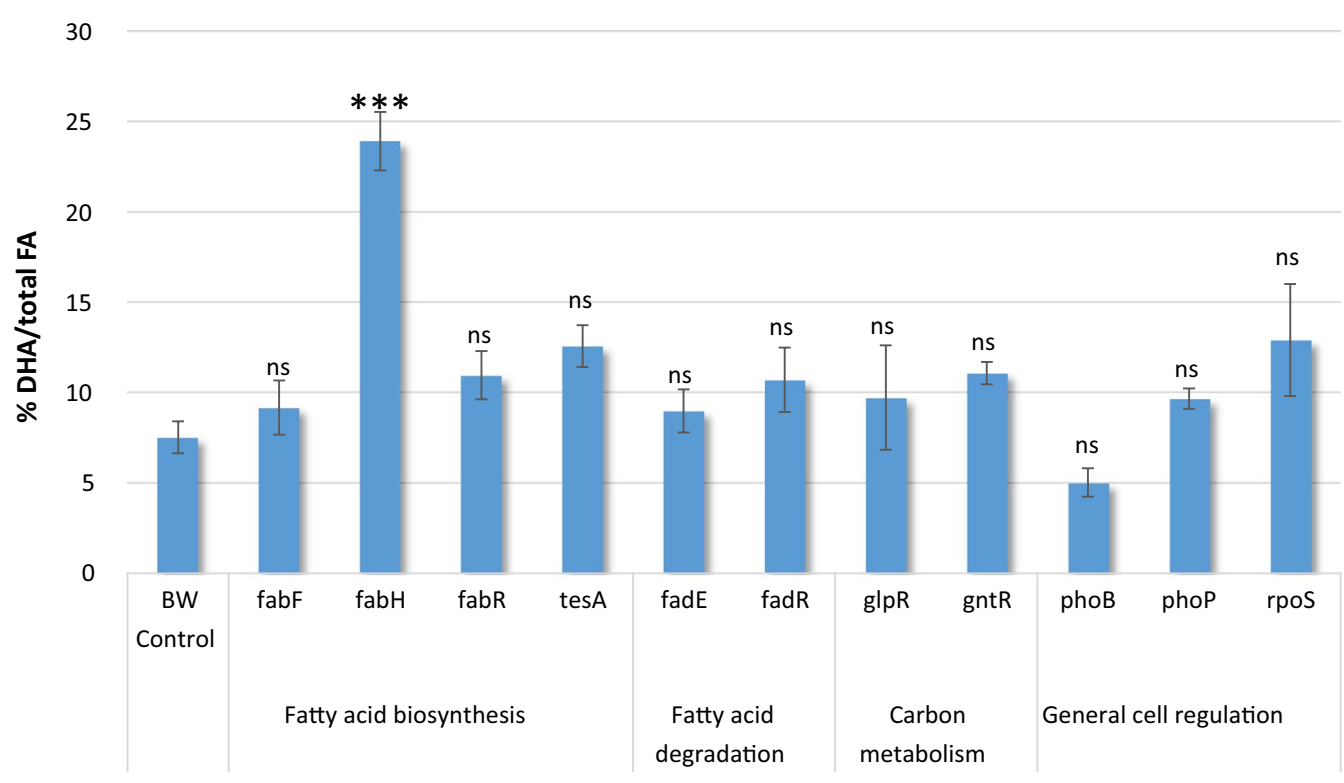

Strains transformed with pDHA4

Fig. 3 DHA production in KEIO mutant strains and reference strain BW27783 transformed with pDHA4 plasmid. Bars indicate the mean and standard deviation of DHA percentage in relation to total FA content. Statistical analysis of each FA content for each strain was performed for at least three replicates using One-Way Anova. Asterisks indicate the degree of significance ${ }^{* * *} p<0.0001$; ns not significant $\left.p>0.05\right)$

Table 3 Selected mutants from KEIO collection [16] for improvement of DHA production by expression of pDHA4 plasmid

\begin{tabular}{lll}
\hline Gene name & Gene code & Protein \\
\hline fabF & b1095 & Ketoacyl synthase II \\
fabH & b1091 & Ketoacyl synthase III \\
fabR & b3963 & Fatty acid synthesis repressor \\
$t e s A$ & b0494 & Acyl-CoA thioesterase I \\
fadE & b0221 & Acyl-CoA dehydrogenase \\
$f a d R$ & b1187 & Global lipid metabolism regulator \\
glpR & b3423 & Glycerol-3-phosphate transcriptional repressor \\
gntR & b3438 & Entner-Doudoroff pathway transcriptional repressor \\
phoB & b0399 & Response factor. Phosphate dual regulation factor \\
phoP & b1130 & Response factor. Phosphate dual regulation factor \\
$r p o S$ & b2741 & Sigma factor. Represses of fadA, fadB, fadE and fadH \\
\hline
\end{tabular}


only increased DHA production to $9.1 \%$, a less prominent effect than the dual partial inhibition of FabB and FabF produced by cerulenin (17\%) shown previously.

ii. Two genes coding for enzymes involved in the FA degradation pathway, in order to avoid DHA consumption: $f a d E$ (FA $\beta$-oxidation enzyme), fadR (FA metabolism regulator). None of these two mutants showed a significant difference in the DHA content compared to BW-pDHA4, since $\triangle f a d E$-pDHA4 and $\triangle f a d R$-pDHA4 had a DHA content of 8.9 and $10.7 \%$ respectively.

iii. Deletion of gene regulation factors involved in carbon metabolism pathways: $g l p R$ (repressor of the glycerol-3-phosphate regulon) and gnt $R$ (repressor of the Entner-Doudoroff pathway). Both mutants did not have a significant difference in DHA content compared to the BW-pDHA4, from 7.5 to $9.8 \%$ in $\Delta g l p R$-pDHA4 and $11.0 \%$ in $\Delta g n t R$-pDHA4.

iv. Three genes coding for general regulation proteins involved in the FA metabolic switch between biosynthesis and degradation [20]: phoB and phoP (dual regulator involved in FA regulation network) and rpoS (sigma factor involved in FA regulation network). The regulation mutants did not show a significant difference in the DHA content compared to BW-pDHA4, since $\Delta p h o B$-pDHA4, $\Delta p h o P$-pDHA4 and $\triangle r p o S$-pDHA4 had a 5.0, 9.6 and $12.9 \%$ of DHA.

\section{Discussion}

The main goal in this work was to obtain an E. coli strain with the ability to produce higher amounts of DHA, avoiding the addition of exogenous expensive compounds such as cerulenin to the culture. Previous studies [9-11] suggested that there is substrate competition between FAS and Pfa biosynthesis pathways, since Claisen condensation of malonyl-ACP and acetyl-CoA initiates both synthetic pathways. Sublethal concentrations of cerulenin partially alter the KS activity of FabB and FabF in FAS system, which increases the concentration of the intracellular malonyl-CoA pool available for the heterologous DHA pathway [15]. Furthermore, it has been previously reported [11] that the heterologous Pfa system from Thraustochytrium sp. expressed in E. coli was not inhibited by cerulenin, while the FAS system was indeed affected. Thus, the Pfa pathway seems to use the intracellular malonyl-CoA pool for DHA production, which increases when conventional FA synthesis (via the $f a b$ pathway) is partially blocked.

In fact, our in vivo results showed that DHA production is certainly improved in strain $\triangle f a b H$-pDHA4 (Fig. 2), even when cerulenin is not present in the medium, which could indicate that the availability of
malonyl-CoA is actually a bottleneck for DHA production in our system. Thus, as shown in the model we present in Fig. 4, our data suggests the existence of a substrate competition mechanism between the FA synthase systems FAS and Pfa, analogous to what happens in the case of cerulenin inhibition.

In fatty acid synthesis, substrates bound to ACP are processed by three different KS. In the first cycle of elongation, FabH (KS III) condenses the substrates malonyl-ACP and acetyl-CoA to produce acetoacetyl-ACP. Afterwards, FabB (KS I) plays the essential role of elongating the primer acetoacetyl-ACP to $16-\mathrm{C}$ long FA and elongate unsaturated fatty acids. Furthermore, in vitro studies found that FabB and FabF are also capable of using acetyl-ACP as a substrate by a side reaction in which malonyl-ACP is decarboxylated to acetyl-ACP, and then used for Claisen condensation with a malonyl-ACP residue [21]. Although this hypothesis is based on an in vitro assay, it could explain the residual FA synthetic activity in the strain carrying $f a b H$ deletion. Furthermore, it has been observed that a $\triangle f a b H$ Lactobacillus lactis strain still retained 10\% FA activity [22], probably due to side initiation KS reactions carried out by FabB and FabF [23].

Finally, last elongation cycle in E. coli is catalysed by FabF (KS II), adding two carbons to palmitoleic acid to produce cis-vaccenic acid [20]. This subsequent activity of the three ketosynthases could explain why the deletion of the main initial condensation enzyme of FA biosynthesis seems to have an even larger impact on DHA production than the inhibition caused by cerulenin, since FabH catalyses an earlier step during biosynthesis than FabB and FabF. Furthermore, the secondary catalytic ability of FabB is observed in $\triangle f a b H$ strain treated with cerulenin, which shows a higher level of DHA, meaning the levels of intracellular malonyl-CoA is even higher.

Additionally, FabH has been reported as essential in strains lacking spoT [22]; however, in wild type strains, it has been proven to not be essential [16, 24]. As showed in Fig. 2c, the deletion of $f a b H$ has an impact on cell growth, same way the inhibition of cerulenin on FabB/FabF activity does.

Interestingly, deletion of $f a b F$ did not have a noteworthy effect on DHA content. This fact could be explained by the subsequent activity of the ketosynthase enzymes explained previously, since the cell is able to generate long-chain FAs by FabB activity, and therefore, FabF activity is not essential. In summary, these data indicate that, in order to increase the intracellular malonyl-CoA pool, it is necessary to intercept the metabolic route at the initial condensation steps of FA synthesis, or even earlier, at acetyl-CoA carboxilase (ACC) or FabD reactions. However, FabH is the 


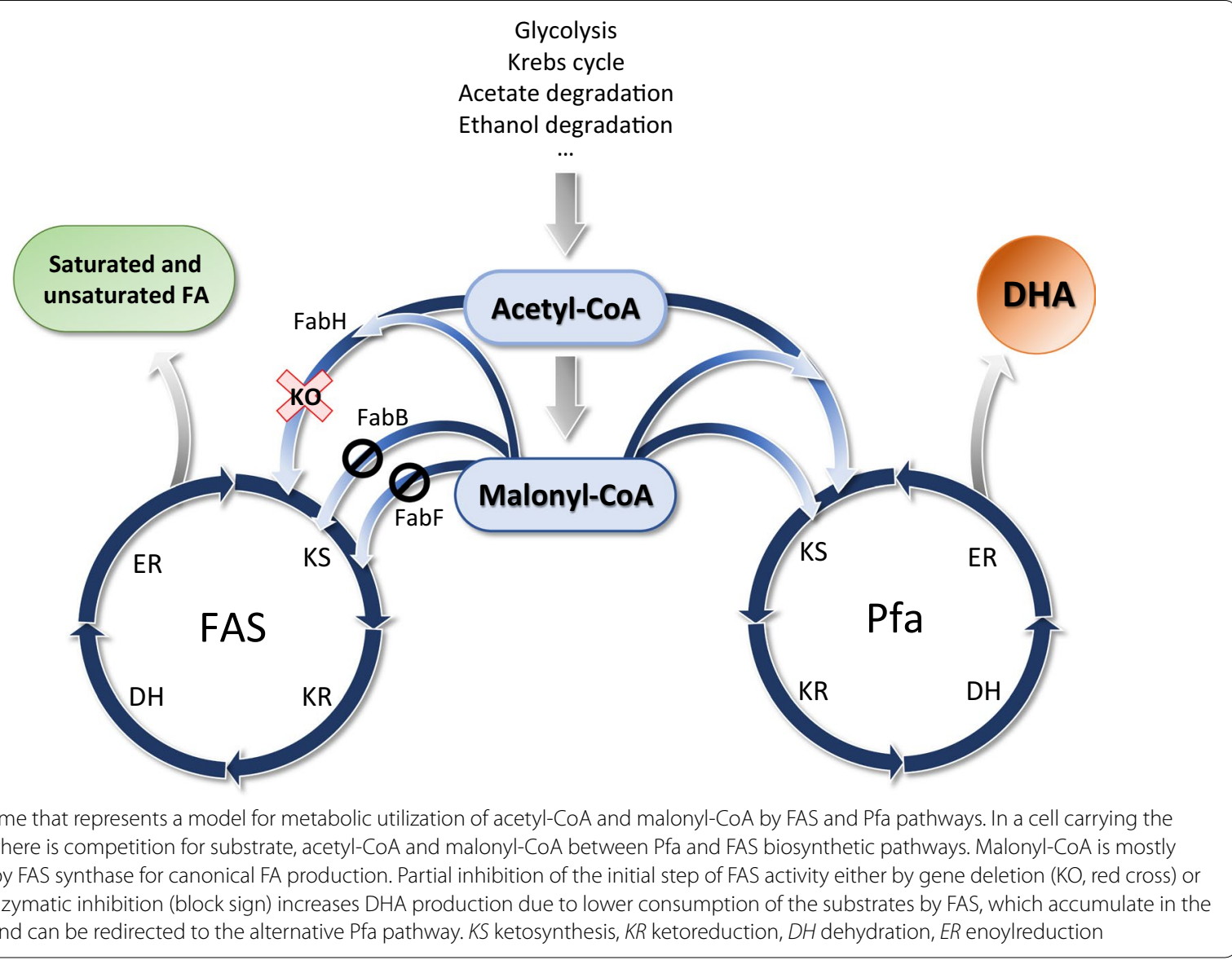

only non-essential gene at this stage of FA synthesis, making it a good starting point for malonyl-CoA intracellular accumulation. Further analysis of other KEIO mutants shows that genes implicated in the regulation of FA metabolism had no significant improvement in DHA production. First, the transcription factors FabR, FadR and RpoS, as well as PhoB and PhoP, coordinate the biosynthesis and degradation of FA according to the energetic requirements of the cell [20]. It would be expected that elimination of the positive regulators of FA biosynthesis led to a significant increase of DHA. Nevertheless, no improvement in DHA production was observed. Thus, we could only speculate that the tight regulation of the FA metabolic pathways is exerted by modulation of the key enzymes of the cycle in order to respond to the energetic status of the cell [25].

Furthermore, we selected the enzyme from $\beta$-oxidation pathway FadE, with the aim of reducing FA degradation and, thus, avoiding DHA catabolism. However, this deletion had no impact on DHA accumulation. This observation was shocking since several studies reported that deletion of $f a d E$ and other genes of the $\beta$-oxidation pathway do reduce FA degradation, and therefore, increase FA accumulation [20].

\section{Conclusions}

The main conclusion of this work is that deletion of $\mathrm{fabH}$ in a LC-PUFA3 producer organism, which carries a Pfa synthase, improves DHA production by diminishing substrate competition with FAS. Furthermore, this approach could be applied to other metabolic routes, which share malonyl-CoA as common precursor with FA synthesis, such as polyketide synthases, and other metabolites.

This approximation is a proof of concept that shows the potential of altering carbon fluxes towards a heterologous pathway.

\section{Methods}

Bacterial cells and cultivation

Escherichia coli BW27783 (Table 1) was transformed with plasmid pDHA4 [7] to be used as host for the 
recombinant $p f a$ genes. Bacterial cells were inoculated from an overnight culture at $37{ }^{\circ} \mathrm{C}$ grown to saturation. Cultures at an initial $\mathrm{OD}=0.15$ were incubated on a rotary shaker $(170 \mathrm{rpm})$ at $15{ }^{\circ} \mathrm{C}$ for $72 \mathrm{~h}$ in $\mathrm{LB}$ medium (Conda laboratories). When appropriate, kanamycin $(50 \mu \mathrm{g} / \mathrm{ml}$, Apollo Scientific) or chloramphenicol $(25 \mu \mathrm{g} / \mathrm{ml}$, Apollo Scientific) were added to the medium. In order to test the effect of cerulenin on cells, cultures were treated with a range of cerulenin concentrations $(0-2 \mu \mathrm{g} / \mathrm{ml}$, Cayman chemicals). Growth was determined by measuring the optical density at $600 \mathrm{~nm}$ (OD600) in a spectrophotometer (Nanodrop2000c, Nanodrop). Cells were collected by centrifugation in a Eppendorff $5810 \mathrm{R}$ centrifuge at $1700 \times g$ for $10 \mathrm{~min}$ at room temperature.

The growth curve of each strain was measured by OD at $600 \mathrm{~nm}$ in a Victor 3 system (Perkin Elmer) using 24-well plates with a culture volume of $750 \mu \mathrm{l}$. Each data point for the curve was calculated by the average of 12 independent cultures, and the maximum doubling time was calculated at early exponential phase.

Selected in frame mutants from KEIO collection [16] are summarized in Table 3 . The strains were transformed with plasmid pDHA4 by electroporation, and checked for chloramphenicol resistance on LB agar plates $(1.5 \%$ (wt/v) agar; Conda laboratories) Gene deletion of $f a b H$ was checked by PCR using primers FabHS (5'-GCTACA AAAGAGCCTGACGAGG) and FabHE (5'-CAATCA CACCAGCGCAAACCAG), and primers for kanamycin resistance insertion Rk1 (5'-AGCCGAATAGCCTCT CCACCCA) and Fk2 (5'-CATCTCACCTTGCTCCTG CCGA) as described previously [18].

\section{Fatty acid analysis}

FA composition and DHA quantitation were carried out by gas chromatography at Biomar Microbial Technologies (León, Spain) using pelleted cells. FAs were isolated from the samples following the Folch method [26]. The esterification with methanol was performed as described previously [27]. Concentrated FAMEs were analyzed on a gas chromatograph (model 7890A, Agilent) equipped with a capillary column DB-23 and flame ionization detection using helium as a carrier gas at 52.248 psi constant pressure. Temperature was kept at $130^{\circ} \mathrm{C}$ for $1 \mathrm{~min}$, then it was increased to $215^{\circ} \mathrm{C}$ at a $2.75^{\circ} \mathrm{C} / \mathrm{min}$ ratio, and finally it was maintained at $215^{\circ} \mathrm{C}$ for $12 \mathrm{~min}$. The column was then cleaned by a ballistic increase of temperature to $230{ }^{\circ} \mathrm{C}$ and kept at this temperature for $2 \mathrm{~min}$. The sample was injected at $270{ }^{\circ} \mathrm{C}$ in a split-splitless chamber with a split ratio of 10:1. The compounds were detected in a flame ionization detector (FID) at $280^{\circ} \mathrm{C}$ with the following gas flows: air $(200 \mathrm{ml} / \mathrm{min}), \mathrm{H}_{2}(30 \mathrm{ml} / \mathrm{min})$, and
$\mathrm{N}_{2}(22.5 \mathrm{ml} / \mathrm{min})$. FAMEs were identified by comparing the retention time with commercial standard Supelco37 Component FAME Mix (Sigma Aldrich). Quantitation of DHA was determined using commercial standards. Methyl tricosanoate was used as internal standard.

\section{Statistical analysis}

The statistical analysis of the gas chromatography results was made using IBM SPSS Statistics version 22.0. (IBM Corporation). Comparison of group means against the control group was performed individually for each FA by the One-Way ANOVA software using Dunnet post hoc analysis. The significance value was set at 0.05 .

\section{Additional file}

Additional file 1. Additional Figures S1 and S2.

Authors' contributions

LGR and BL conceived and designed experiments. LGR performed the experiments and performed data collection. LGR, GM and FC wrote the manuscript. All authors read and approved the final manuscript.

\section{Acknowledgements}

We are grateful to Dr. Antonio Fernández and Dr. Francisco Romero for GC analysis performed at Biomar Microbial Technologies, León, Spain. We acknowledge Dr. Yoshitake Orikasa from Obihiro University of Agriculture and Veterinary Medicine (Japan) for the generous gift of plasmid pDHA4. We are also grateful to the anonymous reviewers for their valuable comments.

\section{Competing interests}

The authors declare that they have no competing interests.

Availability of data and materials

All the data analysed during this study have been included in this published article and additional data.

Consent for publication

Not applicable.

Ethics approval and consent to participate Not applicable.

\section{Funding}

The work in the FdIC and GM laboratories was financed by the Spanish Ministry of Economy, Industry and Competitiveness Grant BFU2014-55534-C2.

\section{Publisher's Note}

Springer Nature remains neutral with regard to jurisdictional claims in published maps and institutional affiliations.

Received: 12 February 2018 Accepted: 11 May 2018

Published online: 08 June 2018

\section{References}

1. Swanson D, Block R, Mousa SA. Omega-3 fatty acids EPA and DHA: health benefits throughout life. Adv Nutr. 2012;3:1-7.

2. Lee JH, O'Keefe JH, Lavie CJ, Marchioli R, Harris WS. Omega-3 fatty acids for cardioprotection. Mayo Clin Proc. 2008;83:324-32. 
3. Astrup AV, Bazinet R, Brenna T, Calder PC, Crawford MA, Dangour A, et al. Fats and fatty acids in human nutrition. Report of an expert consultation. 2008.

4. Li D, Hu X. Fish and its multiple human health effects in times of threat to sustainability and affordability: are there alternatives? Asia Pac J Clin Nutr. 2009;18:553-63.

5. Abedi E, Sahari MA. Long-chain polyunsaturated fatty acid sources and evaluation of their nutritional and functional properties. Food Sci Nutr. 2014:2:443-63.

6. Shulse CN, Allen EE. Widespread occurrence of secondary lipid biosynthesis potential in microbial lineages. PLoS ONE. 2011;6:e20146.

7. Orikasa Y, Tanaka M, Sugihara S, Hori R, Nishida T, Ueno A, et al. pfaB products determine the molecular species produced in bacterial polyunsaturated fatty acid biosynthesis. FEMS Microbiol Lett. 2009;295:170-6.

8. Metz JG, Kuner J, Rosenzweig B, Lippmeier JC, Roessler P, Zirkle R. Biochemical characterization of polyunsaturated fatty acid synthesis in Schizochytrium: release of the products as free fatty acids. Plant Physiol Biochem. 2009:47:472-8.

9. Morita N, Nishida T, Tanaka M, Yano Y, Okuyama H. Enhancement of polyunsaturated fatty acid production by cerulenin treatment in polyunsaturated fatty acid-producing bacteria. Biotechnol Lett. 2005;27:389-93.

10. Wan X, Peng Y-F, Zhou X-R, Gong Y-M, Huang F-H, Moncalián G. Effect of cerulenin on fatty acid composition and gene expression pattern of DHA-producing strain Colwellia psychrerythraea strain $34 \mathrm{H}$. Microb Cell Fact. 2016;15:30.

11. Hauvermale A, Kuner J, Rosenzweig B, Guerra D, Diltz S, Metz JG. Fatty acid production in Schizochytrium sp.: involvement of a polyunsaturated fatty acid synthase and a type I fatty acid synthase. Lipids. 2006:41:739-47.

12. Peng $Y-F$, Chen $W-C$, Xiao K, Xu L, Wang L, Wan X. DHA production in Escherichia coli by expressing reconstituted key genes of polyketide synthase pathway from marine bacteria. PLoS ONE. 2016;11:e0162861.

13. Price AC, Choi KH, Heath RJ, Li Z, White SW, Rock CO. Inhibition of $\beta$-ketoacyl-acyl carrier protein synthases by thiolactomycin and cerulenin: structure and mechanism. J Biol Chem. 2001;276:6551-9.

14. Moche M, Schneider G, Edwards P, Dehesh K, Lindqvist Y. Structure of the complex between the antibiotic cerulenin and its target, beta-ketoacylacyl carrier protein synthase. J Biol Chem. 1999;274:6031-4.
15. van Summeren-Wesenhagen PV, Marienhagen J. Metabolic engineering of Escherichia coli for the synthesis of the plant polyphenol pinosylvin. Appl Environ Microbiol. 2015;81:840-9.

16. Baba T, Ara T, Hasegawa M, Takai Y, Okumura Y, Baba M, et al. Construction of Escherichia coli K-12 in-frame, single-gene knockout mutants: the Keio collection. Mol Syst Biol. 2006;2006(2):0008.

17. Guzman LM, Belin D, Carson MJ, Beckwith J. Tight regulation, modulation, and high-level expression by vectors containing the arabinose $p(B A D)$ promoter. J Bacteriol. 1995;177:4121-30.

18. Datsenko KA, Wanner BL. One-step inactivation of chromosomal genes in Escherichia coli K-12 using PCR products. Proc Natl Acad Sci USA. 2000;97:6640-5.

19. Khlebnikov A, Datsenko KA, Skaug T, Wanner BLB, Keasling JJD. Homogeneous expression of the pBAD promoter in Escherichia coli by constitutive expression of the low-affinity high-capacity araE transporter. Microbiology. 2001;147:3241-7.

20. Janßen HJ, Steinbüchel A. Fatty acid synthesis in Escherichia coli and its applications towards the production of fatty acid based biofuels. Biotechnol Biofuels. 2014;7:7.

21. Alberts A, Bell R, Vagelos P. Acyl carrier protein. XV. Studies of $\beta$-ketoacylacyl carrier protein synthetase. J Biol Chem. 1972;247:3190-8.

22. Lai CY, Cronan JE. $\beta$-Ketoacyl-acyl carrier protein synthase III $(\mathrm{FabH})$ is essential for bacterial fatty acid synthesis. J Biol Chem. 2003;278:51494-503.

23. Cronan JE, Thomas J. Bacterial fatty acid synthesis and its relationships with polyketide synthetic pathways. Methods Enzymol. 2009:459:395-433.

24. Yao Z, Davis RM, Kishony R, Kahne D, Ruiz N. Regulation of cell size in response to nutrient availability by fatty acid biosynthesis in Escherichia coli. Proc Natl Acad Sci. 2012;109:E2561-8.

25. My L, Rekoske B, Lemke JJ, Viala JP, Gourse RL, Bouveret E. Transcription of the Escherichia coli fatty acid synthesis operon fabHDG is directly activated by FadR and inhibited by ppGpp. J Bacteriol. 2013;195:3784-95.

26. Folch J, Lees M, Sloane Stanley GH. A simple method for the isolation and purification of total lipides from animal tissues. J Biol Chem. 1957;226:497-509.

27. Ichihara K, Fukubayashi Y. Preparation of fatty acid methyl esters for gasliquid chromatography. J Lipid Res. 2010;51:635-40.
Ready to submit your research? Choose BMC and benefit from:

- fast, convenient online submission

- thorough peer review by experienced researchers in your field

- rapid publication on acceptance

- support for research data, including large and complex data types

- gold Open Access which fosters wider collaboration and increased citations

- maximum visibility for your research: over $100 \mathrm{M}$ website views per year

At BMC, research is always in progress.

Learn more biomedcentral.com/submissions 